\title{
INFORMAÇÕES QUE ANTECEDERAM O DIAGNÓSTICO DE TUBERCULOSE PULMONAR E TEMPO DECORRIDO ATÉ O INICIO DO TRATAMENTO EM PACIENTES MATRICULADOS EM CENTRO DE SAÚDE, SÃO PAULO (BRASIL)
}

José Roberto Pretel Pereira Job* José Otávio Alquezar Gozzano* Odilon Ribeiro Bernardes Júnior* * Regina Helena Garcia**

Otávio José Castanha Miralhes**

Marco Aurélio Perosa de Miranda**

\begin{abstract}
JOB, J.R.P.P, et al. Informaçōes que antecederam o diagnóstico de tube*culose pulmonar e tempo decorrido até o início do tratamento em pacientes matriculados em Centro de Saúde. São Paulo (Brasil). Rev. Saúde públ., S. Paulo. 20:21-5. 1986.
\end{abstract}

RESUMO: Em 50 doentes matriculados para tratamento de tuberculose pulmonar no Centro de Saúde I de Sorocaba, SP (Brasil), foram estudadas as informaçōes que antecederam ao diagnóstico da doença tais como: sintomatologia, hábitos, procedência, número de vezes que procurou auxílio médico, conhecimento de contágio prévio e sintomatologia da doença, tempo decorrido entre o início da sintomatologia e o início do tratamento, entre a apresentação ao posto de saúde e o início da terapêutica. Notou-se a importância da sintomatologia respiratória, a demora da procura do médico apesar dos sintomas, a falta de informações do doente a respeito da enfermidade. Apesar do bom desempenho técnico do pessoal médico e paramédico do centro de saúde, constatou-se demora acentuada para a detecção dos casos na população, e, conseqüentemente terapêutica.

UNITERMOS: Tuberculose pulmonar, tratamento. Tuberculose pulmonar, prevenção e controle. Centros de Saúde, administraçĩo.

\section{INTRODUÇÃO}

A tuberculose pulmonar continua a ser considerada como um problema de saúde pública em várias áreas do mundo, inclusive no Brasil ${ }^{\circ}$. O crescimento pupulacional em ritmo superior ao desenvolvimento dos recursos de saúde, a dispersão da população pelos subúrbios e zonas marginais dos grandes centros e a angustiosa situação para o sustento individual e familiar, aliados ao baixo padrão sócio-econômico e cultural, fazem com que se torne dificultosa a detecção e possível tratamento de várias doenças dentre as quais a tuberculose.
Todo programa de saúde pública, especialmente o de controle da tuberculose, visa inicialmente à quebra da cadeia de transmissão da doença, pois sabe-se que cada doente não descoberto tende a infectar de 10 a 15 pessoas em um ano, sendo que uma ou duas adquirem a patologia, fazendo com que a mesma permaneça na população como endemia ${ }^{3,6}$.

Quanto mais rápido for o diagnóstico, mais precocemente se poderá iniciar a quimioterapia específica, conseguindo-se assim, além deste, dois outros objetivos,

\footnotetext{
- Do Centro de Ciências Médicas e Biológicas de Sorocaba da Pontificia Universidade Católica de São Paulo - Praça José Ermírio de Moraes. 290 - 18.100 - Sorocaba, SP - Brasil

* Acadêmico do Centro de Ciências Médicas e Biológicas de Sorocaba da Pontifícia Universidade Católica de São Paulo.
} 
JOB, J.R.P.P. et al. Informações que antecederam o diagnóstico de tuberculose pulmonar e tempo decorrido até $o$ início do tratamento em pacientes matriculados em Centro de Saúde, São Paulo (Brasil). Rev. Saúde públ., S. Paulo, 20: 21-5, 1986.

também importantes que são: a diminuição das seqüelas da doença e a manutenção do indivíduo como força de trabalho atuante.

Sabe-se que a quimioterapia suprime a contagiosidade da doença nas primeiras duas semanas, em 80 a $95 \%$ dos $\operatorname{casos}^{10}$. A fim de que um programa de saúde pública funcione, Acuna e col. ${ }^{1}$ salientam dois aspectos fundamentais: a rapidez com que os serviços podem diagnosticar e iniciar o tratamento específico.

Quanto maior o tempo decorrido no regresso do paciente para a confirmação do diagnóstico, maior a percentagem de enfermos que não se matriculam para a terapêutica.

Objetivando fornecer subsídios para o aperfeiçoamento na detecção de casos e de contagiantes propusemo-nos, dentre outros ítens, a colher informações que antecederam ao diagnóstico, e também o tempo decorrido, entre este e o início da medicação específica.

\section{MATERIAL E METODOS}

No período compreendido entre $11 / 5$ / 84 e $11 / 12 / 84$ foram entrevistados 50 pacientes de ambos os sexos, portadores do diagnóstico de tuberculose pulmonar ativa que foram encaminhados ou que espontaneamente procuraram o Centro de Saúde I do Departamento Regional de Saúde de Sorocaba. Dentre este, 9 eram do sexo feminino e 41 do sexo masculino. A idade variava entre 18 e 75 anos.

Todos responderam individualmente as seguintes perguntas: sintomatologia antes do diagnóstico, tempo decorrido entre o início dos sintomas e a procura de assistência médica, contágio prévio, conhecimento dos sintomas da doença, serviço médico procurado inicialmente, hábito, procedência, número de vezes que procurou o serviço médico até a orientação do diagnóstico, tempo decorrido entre o início dos sintomas e o início da quimioterapia, e tempo decorrido entre o diagnóstico laboratorial e o tratamento.

\section{RESULTADOS}

Em relação à sintomatologia prévia ao diagnóstico, 44 (88\%) doentes referiram tosse, $43(86 \%)$ febre, 37 (74\%) sudorese noturna, 34 (68\%) expectoração, emagrecimento em 33 (66\%), dispnéia em 28 $(56 \%)$, dor no tórax em $25(50 \%)$ e hemoptise em 16 (32\%).

Considerando-se o tempo decorrido entre o início da sintomatologia e a procura do serviço médico observamos que 17 (34\%) doentes demoraram menos do que um mês para fazê-lo, $16(32 \%)$ entre 1 e 2 meses, $8(16 \%)$ entre 2 e 3 , e 9 $(18 \%)$ três ou mais meses.

Onze pacientes (22\%) referiram contágio anterior, com doentes conhecidos, enquanto $39(78 \%)$ desconheciam tal contágio.

Quanto ao ítem conhecimento prévio da sintomatologia, 17 (34\%) o sabiam, enquanto que $33(66 \%)$ o negaram.

Dezenove pacientes referiram alcoolismo e tabagismo como hábitos, conjuntamente, correspondendo a $38 \%$, sendo que outros 19 eram só fumantes, $9(18 \%)$ só etilistas e apenas $3(6 \%)$ negaram qualquer tipo de hábito.

Da zona urbana procediam 43 doentes $(86 \%)$ e apenas $7(14 \%)$ eram da zona rural.

Onze doentes procuraram o médico apenas uma vez (22\%) para a orientação do diagnóstico, $21(42 \%)$ o fizeram duas 
JOB, J.R.P.P, et al. Informações que antecederam o diagnóstico de tuberculose pulmonar e tempo decorrido até o início do tratamento em pacientes matriculados em Centro de Saúde, São Paulo (Brasil). Rev. Saúde públ., S. Paulo, 20: 21-5, 1986.

vezes, $9(18 \%)$ por três vezes, e outros $9(18 \%)$ consultaram-no por quatro ou mais vezes.

O consultório particular foi procurado por $5(10 \%)$ doentes, serviços ambulatoriais por $22(44 \%), 8(16 \%)$ procuraram pronto-socorro e 15 (30\%) postos de saúde.

O tempo decorrido entre o início dos sintomas e o tratamento foi inferior a 30 dias, em 10 doentes (20\%), entre 30 e 60 dias em 10 (20\%), entre 60 e 90 dias em $13(26 \%)$, e mais que 3 meses em 17 , correspondendo a $34 \%$.

Em relação ao tempo decorrido entre a realização do exame e o início do tratamento temos que $21(42 \%)$ doentes o começaram até o $3^{\circ}$ dia após a realização do mesmo, $12(24 \%)$ entre o $3^{\circ}$ e o $6^{\circ}, 11(22 \%)$ entre o $7^{\circ}$ e o $9^{\circ}$, e apenas $6(12 \%)$ gastaram 10 ou mais dias para iniciá-lo, após a apresentação ao posto de saúde.

\section{DISCUSSĀO}

Tosse, febre, sudorese, expectoração, emagrecimento, dispnéia, dor torácica e hemoptise foram, em ordem decrescente, os sintomas mais constantemente relatados. A grande frequiência do sintoma tosse reforça a indicação do estudo baciloscópico de escarro para todos os indivíduos sintomáticos respiratórios como recomendam o Programa Nacional para o Controle da Tuberculose ${ }^{8}$ e a OPAS : Herrera Contrera e Villegas Bobadilla ${ }^{6}$ e Mendez Aguilera ${ }^{7}$ indicam tal pesquisa prioritariamente para aqueles enfermos com tosse e expectoração por mais de 15 dias, especialmente naqueles com mais de 15 anos de idade.

A percentagem de doentes com tosse e expectoração assemelha-se a encontrada por Mendez Aguilera ${ }^{7}$, de $90 \%$, e também a de Gozzano e col. ${ }^{5}$, de $86 \%$.
Emagrecimento esteve presente em $66 \%$ dos indivíduos, diferente da relatada por Gozzano e col. ${ }^{5}$, em pacientes que necessitaram de internação, pois naqueles foi o principal sintoma encontrado.

O tempo decorrido do início dos sintomas e a procura do médico, em $66 \%$ dos doentes, foi maior do que 30 dias. Tal fato é importante porque mostra a presença mais duradoura destes pacientes como fonte transmissora na população e também pelas sequielas que podem lhes advir do maior tempo sem tratamento. Aproximadamente $1 / 3$ dos pacientes procuraram o médico dentro do primeiro mês, percentagem semelhante a encontrada por Acuna e col. ${ }^{1}$ e Gozzano e col. ${ }^{5}$.

Apenas $22 \%$ declararam contágio anterior com portadores de tuberculose e $34 \%$ tinham conhecimento prévio dos sintomas da doença.

De acordo com os dados obtidos por Comstock ${ }^{4}$, Mendez Aguilera ${ }^{7}$ e Gozzano e col. ${ }^{5}$, está a percentagem de doentes que procedem de zona urbana $(86 \%)$ contra $24 \%$ que vem de zona rural, confirmando-se a tendência da tuberculose ocorrer principalmente em áreas metropolitanas.

Dos 50 doentes, apenas 3 não tinham hábitos. Os restantes 47 apresentavam pelo menos um, podendo o álcool, segundo a OPAS ${ }^{2}$, ser considerado como fator predisponente. Rosemberg ${ }^{11}$ explica tal fato por uma provável menor capacidade de defesa pela desnutrição que frequientemente acompanha o etilismo. Os dados acima são semelhantes aos encontrados por Gozzano e col. ${ }^{5}$ em pacientes internados com tuberculose pulmonar.

Em relação ao número de vezes que procurou a assistência médica, $36 \%$ dos doentes com sintomatologia compatível tiveram que procurá-la por três ou mais 
JOB, J.R.P.P. et al. Informações que antecederam o diagnóstico de tuberculose pulmonar e tempo decorrido até o início do tratamento em pacientes matriculados em Centro de Saúde, São Paulo (Brasil). Rev. Saúde públ., S. Paulo, 20: 21-5, 1986.

vezes para a orientação adequada. Tal percentagem é maior que a encontrada por Gozzano e col. ${ }^{5}$, nos internados por tuberculose que foi de $28 \%$.

Os serviços procurados inicialmente foram, em $44 \%$ das vezes, os ambulatórios, $30 \%$ os centros de saúde, $16 \%$ pronto-socorros e $10 \%$ o consultório particular. Tais dados são semelhantes aos encontrados por Gozzano e col. ${ }^{5}$.

Ao observar-se o tempo decorrido entre o início da sintomatologia e o do tratamento nota-se que $80 \%$ dos enfermos tiveram demora maior que um mês e que, em $60 \%$, esta foi maior do que 2 meses. Tais dados são semelhantes aos de Acuna e col. ${ }^{1}$ e Gozzano e col. ${ }^{7}$, mostrando demora considerável na identificação das fontes de contágio para a população e, também, a maior possibilidade de agravamento do quadro clínico pela falta de terapêutica específica.

Oitenta por cento dos doentes iniciaram a quimioterapia em até 9 dias após a apresentação ao posto de saúde para exame baciloscópico, percentagem superior à citada por Senties e Pantle ${ }^{12}$, no ano de 1977 , que foi de $78,4 \%$.

\section{CONCLUSÓES}

A presença de queixas respiratórias foi uma constante nesta casuística ressaltando a grande importância da investigação subsequiente para o diagnóstico etiológico do assim chamado "sintomático pulmonar".
A grande maioria desta população desconhecia pregressamente a sintomatologia da doença. Desta forma, torna-se importante, em termos de saúde pública, uma maior educação acerca do problema, a fim de que seja buscado o auxílio médico, mais rapidamente.

Etilismo e tabagismo aparecem com grande frequiência podendo interferir na eclosão da doença, quiçá por causarem distúrbios imunológicos ou talvez como fatores de agravamento do quadro clínico.

O tempo decorrido para a confirmação do diagnóstico e início da terapêutica específica foi de menos que 10 dias em $88 \%$ dos doentes, sugerindo tal fato um bom desempenho do pessoal médico e paramédico nas rotinas de colheita, leitura do exame e início do tratamento dos pacientes matriculados no serviço.

$O$ intervalo entre o início da sintimatologia e o do tratamento foi maior do que 2 meses, em $60 \%$, sugerindo tal fato que apesar do bom desempenho do pessoal técnico após a chegada do paciente; provavelmente há retardo na motivação do doente para "pedir" saúde, pela demora em procurar os serviços, fato relacionado, talvez, com a ignorância sobre a doença devido à má educação sanitária ou pelas dificuldades de cobertura da área pelos postos de saúde, comprometendo, assim, a possibilidade de um melhor desempenho do programa de controle da tuberculose. 
JOB, J.R.P.P. et al. Informações que antecederam o diı gnóstico de tuberculose pulmonar e tempo decorrido até o início do tratamento em pacientes matriculados em Centro de Saúde, São Paulo (Brasil). Rev. Saúde públ., S. Paulo, 20: 21-5. 1986.

JOB, J.R.P.P et al. [Information relating to health before the diagnosis of pulmonary tuberculosis and time lag before beginning of treatment in patients enrolled at health center in S. Paulo, Brazill. Rev. Saúde públ., S. Paulo, 20:26-32, 1986

ABSTRACT: Pulmonary tuberculosis still remains a serious public health problem in Brazil. The National Tuberculosis Control Program has as its aim the interruption of the chains of transmition by diagnosis and, consequently, by early treatment. It is known that specific chemotherapy reduces the degree of contagion in the first two weeks of treatment. The sooner the health program detects and begins therapy, the bigger average number of patients who will participate in it. With the intention of providing information to increase efficiency in detecting tuberculosis and contagious cases it was collected data relating to the patients' health before diagnosis and also to the time lag between this diagnosis and the beginning of specific treatment. Fifty cut-patients (50) suffering from tuberculosis of the lungs were questioned, during treatment, for information relating to the period proceeding diagnosis. Information sought covered: symptomatology before contamination, place and number of consultations, habits time elapsed between the occurrence of first symptoms and first visit to the physician, lapse of time between the occurrence of first symptoms and the beginning of treatment, knowledge about symptomatology of the disease and lapse of time between the first appearance at the health clinic and the beginning of treatment. The importance of the factors: respiratory symptomatology, the considerable time lapse before looking for a physician and lack of information about the disease was observed. Despite the dedication of the medical and paramedical services there was delay in detecting the sick person within the community.

UNITERMS: Tuberculosis, pulmonary therapy. Tuberculosis, pulmonary, prevention and control. Community health services, administration.

\section{REFERENCIAS BIBLIOGRÁFICAS}

1. ACUNA, D.; FERRER, X.; GALVEZ, B.; MUJICA, L.; MAUREIRA, L.M.; SOTO, A. \& ROJAS, L. Tuberculosis pulmonar: tiempo transcurrido entre inicio de los sintomas respiratorios y el tratamiento. Rev. méd. Chile, 109: $628-33,1981$.

2. BENENSON, A.S., ed. El control de las enfermedades transmisibles en el hombre. 12. ${ }^{\mathrm{a}}$ ed. Washington, D.C., Organizacion Panamericana de la Salud, 1978. (OPAS - Publ, cient., 372).

3. CASTILLO ROBLES, G. \& JIMENEZ, G.T. Epidemiologia de la tuberculosis. Rev. méd. Hosp. gen., México, 45:1647, 1982

4. COMSTOCK, G.W. Epidemiology of tuberculosis. Amer. Rev. resp. Dis. 125 (3 pt. 2):8-15, 1982.

5. GOZZANO, J.O.A.; JOB, J.R.P.P.; BERNARDES JR., O.R.; MIRANDA, M.A.P. \& MIRALHES, O.J.C. Inforformações que antecederam o diagnóstico de tuberculose pulmonar entre doentes internados em hospital especializado. J. Pneumol. 11, 1985 [no prelo].

6. HERRERA CONTRERA, M. \& VILLEGAS BOBADILLA, A, Diagnóstico de la tuberculosis pulmonar. Salud públ. Méx., 24:289-302, 1982.

7. MENDEZ AGUILERA, P. Busqueda de casos. Simposio I Programa nacional de control de la tuberculosis y estado epidemiologico actual. El Tórax, 28:536, 1982

8. MINISTERIO DA SAUDE. Secretaria Nacional de Programas Especiais de Saúde. Divisão Nacional de Pneumologia Sanitária. Manual de normas para o controle de tuberculose. Brasília, 1979.

9. ONISHI, R - Tuberculose pulmonar. Rev. Med., S. Paulo, 64:52-3, 1982.

10. PACHECO, R.C.; OLVERA, R. \& HERRERA, M. Panorama epidemiológico de la tuberculosis en la Republica Mexicana. Salud públ. Méx., 22:251-9, 1980.

11. ROSEMBERG, J. Apostila de epidemiologia da tuberculose. Sorocaba, Centro Acadêmico "Vital Brazil", 1978.

12. SENTIES, R. \& PANTLE, M.E. La celeridad en el diagnóstico de los pacientes tuberculosos. Salud públ. Méx., 21:103-7, 1979.

Recebido para publicação em 31/05/1985. Aprovado para publicação em $21 / 10 / 1985$. 